\title{
Uniform Estimate of the Finite-Time Ruin Probability for All Times in a Generalized Compound Renewal Risk Model
}

\author{
Qingwu Gao, ${ }^{1}$ Na Jin, ${ }^{2}$ and Juan Zheng $^{3}$ \\ ${ }^{1}$ School of Mathematics and Statistics, Nanjing Audit University, Nanjing 211815, China \\ 2 Investment Department, Nanjing Times Media Co., Ltd., Nanjing 210039, China \\ ${ }^{3}$ School of Mathematics and Statistics, Zaozhuang University, Zaozhuang 277160, China
}

Correspondence should be addressed to Qingwu Gao, gaochingwoo@yahoo.com.cn

Received 4 April 2012; Accepted 23 September 2012

Academic Editor: Raymond Chan

Copyright (C) 2012 Qingwu Gao et al. This is an open access article distributed under the Creative Commons Attribution License, which permits unrestricted use, distribution, and reproduction in any medium, provided the original work is properly cited.

We discuss the uniformly asymptotic estimate of the finite-time ruin probability for all times in a generalized compound renewal risk model, where the interarrival times of successive accidents and all the claim sizes caused by an accident are two sequences of random variables following a wide dependence structure. This wide dependence structure allows random variables to be either negatively dependent or positively dependent.

\section{Introduction}

In this section, we will introduce a generalized compound renewal risk model, some common classes of heavy-tailed distributions, and some dependence structures of random variables (r.v.s), respectively.

\subsection{Risk Model}

It is well known that the compound renewal risk model was first introduced by Tang et al. [1], and since then it has been extensively investigated by many researchers, for example, Aleškevičienè et al. [2], Zhang et al. [3], Lin and Shen [4], Yang et al. [5], and the references therein. In the paper, we consider a generalized compound renewal risk model which satisfies the following assumptions. 
Assumption $\mathrm{H}_{1}$

The interarrival times $\left\{\theta_{i}, i \geq 1\right\}$ of successive accidents are nonnegative, identically distributed, but not necessarily independent r.v.s with finite mean $\lambda^{-1}$.

\section{Assumption $\mathrm{H}_{2}$}

The claim sizes and their number caused by $n$th accident are $\left\{X_{i}^{(n)}, i \geq 1\right\}$ and $N_{n}, n \geq 1$, respectively, where $\left\{X_{i}^{(n)}, i \geq 1, n \geq 1\right\}$ are nonnegative and identically distributed r.v.s with common distribution $F$ and finite mean $\mu$, and $\left\{X_{i}^{(n)}, i \geq 1\right\}$ are not necessarily independent r.v.s, but $\left\{X_{i}^{(n)}, i \geq 1\right\}$ and $\left\{X_{i}^{(m)}, i \geq 1\right\}$ are mutually independent for all $n \neq m, n, m \geq 1$, while $\left\{N_{n}, n \geq 1\right\}$ are independent, identically distributed (i.i.d.), and positive integer-valued r.v.s with common distribution $G$ and finite mean $v$.

\section{Assumption $\mathrm{H}_{3}$}

The sequences $\left\{\theta_{i}, i \geq 1\right\},\left\{X_{i}^{(n)}, i \geq 1, n \geq 1\right\}$, and $\left\{N_{n}, n \geq 1\right\}$ are mutually independent.

Denote the arrival times of the $n$th accident by $\tau_{n}=\sum_{i=1}^{n} \theta_{i}, n \geq 1$, which can form a nonstandard renewal counting process

$$
N(t)=\sup \left\{n \geq 1, \tau_{n} \leq t\right\}, \quad t \geq 0,
$$

with mean function $\lambda(t)=E N(t)$. Hence the total claim amount at time $\tau_{n}$ and the total claim amount up to time $t \geq 0$ are, respectively,

$$
S_{N_{n}}^{(n)}=\sum_{i=1}^{N_{n}} X_{i}^{(n)}, \quad S(t)=\sum_{n=1}^{N(t)} S_{N_{n}{ }^{\prime}}^{(n)}
$$

and then the insurer's surplus process is given by

$$
R(t)=x+c t-S(t), \quad t \geq 0,
$$

where $x \geq 0$ is the initial surplus and $c>0$ is the constant premium rate. The finite-time ruin probability within time $t>0$ is defined as

$$
\psi(x, t)=P\left(\inf _{0 \leq s \leq t} R(s)<0 \mid R(0)=x\right) .
$$

Clearly, the ruin can only arise at the times $\tau_{n}, 1 \leq n \leq N(t)$, then

$$
\psi(x, t)=P\left(\max _{0 \leq k \leq N(t)} \sum_{n=1}^{k}\left(S_{N_{n}}^{(n)}-c \theta_{n}\right)>x\right) .
$$


Let $\tau$ be a nonnegative r.v., the random time ruin probability is

$$
\psi(x, \tau)=P\left(\max _{0 \leq k \leq N(\tau)} \sum_{n=1}^{k}\left(S_{N_{n}}^{(n)}-c \theta_{n}\right)>x\right) .
$$

In order for the ultimate ruin not to be certain, we assume the safety loading condition holds, namely,

$$
\kappa=c \lambda^{-1}-v \mu>0
$$

In the generalized compound renewal risk model above, if all the sequences $\left\{\theta_{i}, i \geq 1\right\}$, $\left\{X_{i}^{(n)}, i \geq 1, n \geq 1\right\}$, and $\left\{N_{n}, n \geq 1\right\}$ are i.i.d. r.v.s, then the model is reduced to the standard compound renewal risk model introduced by Tang et al. [1], if $N_{1}=N_{2}=\cdots=1$, then the model is the renewal risk model, see Tang [6], Leipus and Šiaulys [7], Yang et al. [8], and Wang et al. [9], among others.

\subsection{Heavy-Tailed Distribution Classes}

We now present some common classes of heavy-tailed distributions. Firstly, we introduce some notions and notation. All limit relationships in the paper are for $x \rightarrow \infty$ unless mentioned otherwise. For two positive functions $a(\cdot)$ and $b(\cdot)$, we write $a(x) \lesssim b(x)$ if $\limsup a(x) / b(x) \leq 1$, write $a(x) \gtrsim b(x)$ if $\liminf a(x) / b(x) \geq 1$, write $a(x) \sim b(x)$ if both, write $a(x)=o(b(x))$ if $\lim a(x) / b(x)=0$. For two positive bivariate functions $a(\cdot, \cdot)$ and $b(\cdot, \cdot)$, we say that relation $a(x, t) \sim b(x, t)$ holds uniformly for all $t \in \Delta \neq \emptyset$ if

$$
\limsup _{x \rightarrow \infty}\left|\frac{a(x, t)}{b(x, t)}-1\right|=0 .
$$

For a distribution $V$ on $(-\infty, \infty)$, denote its tail by $\bar{V}(x)=1-V(x)$, and its upper and lower Matuszewska indices by, respectively, for $y>1$,

$$
J_{V}^{+}=-\lim _{y \rightarrow \infty} \frac{\log \bar{V}_{*}(y)}{\log y}, \quad J_{V}^{-}=-\lim _{y \rightarrow \infty} \frac{\log \bar{V}^{*}(y)}{\log y}
$$

where $\bar{V}_{*}(y)=\liminf \bar{V}(x y) / \bar{V}(x)$ and $\bar{V}^{*}(y)=\lim \sup \bar{V}(x y) / \bar{V}(x)$.

Chistyakov [10] introduced an important class of heavy-tailed distributions, the subexponential class. By definition, a distribution $V$ on $[0, \infty)$ belongs to the subexponential class, denoted by $V \in \mathcal{S}$, if

$$
\overline{V^{* 2}}(x) \sim 2 \bar{V}(x)
$$


where $V^{* 2}$ denotes the 2-fold convolution of $V$. Clearly, if $V \in \mathcal{S}$ then $V$ is long tailed, denoted by $V \in \mathcal{L}$ and characterized by

$$
\bar{V}(x+y) \sim \bar{V}(x), \quad \forall y>0
$$

One can easily see that a distribution $V \in \mathcal{L}$ if and only if there exists a function $f(\cdot):[0, \infty) \mapsto$ $[0, \infty)$ such that

$$
f(x) \uparrow \infty, \quad f(x)=o(x), \quad \bar{V}(x \pm f(x)) \sim \bar{V}(x) .
$$

Korshunov [11] introduced a subclass of the class $\mathcal{S}$, the strongly subexponential class, denoted by $\mathcal{S}_{*}$. Say that a distribution $V \in \mathcal{S}_{*}$, if $\int_{0}^{\infty} \bar{V}(y) d y<\infty$ and

$$
\overline{V_{u}^{* 2}}(x) \sim 2 \overline{V_{u}}(x)
$$

holds uniformly for $u \in[1, \infty)$, where $\overline{V_{u}}(x)=\min \left\{1, \int_{x}^{x+u} \bar{V}(y) d y\right\} \mathbf{1}_{\{x \geq 0\}}+\mathbf{1}_{\{x<0\}}$ with $\mathbf{1}_{A}$ an indicator function of set $A$. Feller [12] introduced another important class of heavy-tailed distributions, the dominant variation class, which is not mutually inclusive with the class $\mathcal{L}$. Say that a distribution $V$ on $[0, \infty)$ belongs to the dominant variation class, denoted by $V \in \Phi$, if

$$
\bar{V}^{*}(y)<\infty, \quad \forall y>0
$$

Cline [13] introduced a slightly smaller class of $\perp \cap \Phi$, the consistent variation class, denoted by $\mathcal{C}$. Say that a distribution $V \in \mathcal{C}$ if

$$
\lim _{y \searrow 1} \bar{V}_{*}(y)=1, \quad \text { or equivalently, } \lim _{y \nearrow 1} \bar{V}^{*}(y)=1 .
$$

Specially, the class $\mathcal{C}$ covers a famous class $\mathcal{R}$, called the regular variation class. By definition, a distribution $V \in \mathcal{R}_{-\alpha}$, if there exists some $\alpha>0$ such that

$$
\lim \frac{\bar{V}(x y)}{\bar{V}(x)}=y^{-\alpha}, \quad \forall y>0
$$

It is well known that for the distributions with finite mean, the following inclusion relationships hold properly, namely,

$$
R \subset \mathcal{C} \subset \mathcal{L} \cap \Phi \subset \mathcal{S}_{*} \subset \mathcal{S} \subset \mathcal{L},
$$

see, for example, Cline and Samorodnitsky [14], Klüppelberg [15], Embrechts et al. [16], and Denisov et al. [17]. For more details of heavy-tailed distributions and their applications to finance and insurance, the readers are referred to Bingham et al. [18] and Embrechts et al. [16]. 


\subsection{Wide Dependence Structure}

In this section we will introduce some concepts and properties of a wide dependence structures of r.v.s, which was first introduced by Wang et al. [19] as follows.

Definition 1.1. Say that r.v.s $\left\{\xi_{i}, i \geq 1\right\}$ are widely upper orthant dependent (WUOD), if for each $n \geq 1$, there exists some finite positive number $g_{U}(n)$ such that, for all $x_{i} \in(-\infty, \infty)$, $1 \leq i \leq n$,

$$
P\left(\bigcap_{i=1}^{n}\left\{\xi_{i}>x_{i}\right\}\right) \leq g_{U}(n) \prod_{i=1}^{n} P\left(\xi_{i}>x_{i}\right)
$$

Say that r.v.s $\left\{\xi_{i}, i \geq 1\right\}$ are widely lower orthant dependent (WLOD), if for each $n \geq 1$, there exists some finite positive number $g_{L}(n)$ such that, for all $x_{i} \in(-\infty, \infty), 1 \leq i \leq n$,

$$
P\left(\bigcap_{i=1}^{n}\left\{\xi_{i} \leq x_{i}\right\}\right) \leq g_{L}(n) \prod_{i=1}^{n} P\left(\xi_{i} \leq x_{i}\right) .
$$

Furthermore, $\left\{\xi_{i}, i \geq 1\right\}$ are said to be widely orthant dependent (WOD) if they are both WUOD and WLOD.

The WUOD, WLOD, and WOD r.v.s are collectively called as widely dependent r.v.s. Recall that if $g_{U}(n) \equiv 1$ or $g_{L}(n) \equiv 1$ for each $n \geq 1$ in Definition 1.1 , then $\left\{X_{i}, i \geq 1\right\}$ are negatively upper orthant dependent or negatively lower orthant dependent (NLOD), see Ebrahimi and Ghosh [20] or Block et al. [21]; if $g_{U}(n)=g_{L}(n) \equiv M$ for some constant $M>0$ and each $n \geq 1$ such that the two inequalities in Definition 1.1 both hold, then $\left\{X_{i}, i \geq 1\right\}$ are extended negatively dependent, see Liu [22] and Chen et al. [23]. Obviously, the WUOD and WLOD structures allow a wide range of negative dependence structures among r.v.s, such as extended negative dependence, negatively upper orthant dependence/negatively lower orthant dependence, negative association (see Joag-Dev and Proschan [24]), and even some positive dependence. For some examples to illustrate that the WUOD and WLOD structures allow some negatively and positively dependent r.v.s, we refer the readers to Wang et al. [19]. below.

The following properties for widely dependent r.v.s can be obtained immediately

Proposition 1.2. (1) Let $\left\{\xi_{i}, i \geq 1\right\}$ be WLOD (or WUOD). If $\left\{f_{i}(\cdot), i \geq 1\right\}$ are nondecreasing, then $\left\{f_{i}\left(\xi_{i}\right), i \geq 1\right\}$ are still WLOD (or WUOD); if $\left\{f_{i}(\cdot), i \geq 1\right\}$ are nonincreasing, then $\left\{f_{i}\left(\xi_{i}\right), i \geq 1\right\}$ are WUOD (or WLOD).

(2) If $\left\{\xi_{i}, i \geq 1\right\}$ are nonnegative and WUOD, then for each $n \geq 1$,

$$
E \prod_{i=1}^{n} \xi_{i} \leq g_{U}(n) \prod_{i=1}^{n} E \xi_{i}
$$


Particularly, if $\left\{\xi_{i}, i \geq 1\right\}$ are WUOD, then for each $n \geq 1$ and any $s>0$,

$$
E \exp \left\{s \sum_{i=1}^{n} \xi_{i}\right\} \leq g_{U}(n) \prod_{i=1}^{n} E \exp \left\{s \xi_{i}\right\} .
$$

Following the wide dependence structures as above, we will consider a generalized compound renewal risk model satisfying Assumption $\mathrm{H}_{3}$ and the following specific assumptions.

Assumption $\mathrm{H}_{1}^{*}$

The interarrival times $\left\{\theta_{i}, i \geq 1\right\}$ are nonnegative, identically distributed and WLOD r.v.s with finite mean $\lambda^{-1}$.

\section{Assumption $\mathrm{H}_{2}^{*}$}

The claim sizes caused by $n$th accident $\left\{X_{i}^{(n)}, i \geq 1\right\}$ are nonnegative, identically distributed and WUOD r.v.s, and the other statements of Assumption $\mathrm{H}_{2}$ are still valid.

The rest of this work is organized as follows: in Section 2 we will state the motivations and main results of this paper after presenting some existing results, and in Section 3 we will give some lemmas and then prove the main results.

\section{Main Results}

In this section, we will present our main results of this paper. Before this, we prepare some related results and the motivations of the main results. For later use, we define $\Lambda=\{t: \lambda(t)>$ $0\}=\left\{t: P\left(\tau_{1} \leq t\right)>0\right\}$.

\subsection{Related Results and Motivations}

As mentioned above, the asymptotics for the finite-time ruin probability in the compound renewal risk model have been studied by many authors. Among them, Aleškevičienè et al. [2] considered the standard compound renewal risk model with condition (1.7) and showed that

(i) if $G \in \mathcal{C}, \bar{F}(x)=o(\bar{G}(x))$ and $E \theta_{1}^{p}<\infty$ for some $p>J_{G}^{+}+1$, then it holds uniformly for all $t \in \Lambda$ that

$$
\psi(x, t) \sim \frac{1}{\mathcal{K}} \int_{x}^{x+\kappa \lambda(t)} G\left(\frac{s}{\mu}\right) d s
$$

(ii) if $F \in \mathcal{C}, \bar{G}(x)=o(\bar{F}(x))$ and $E \theta_{1}^{p}<\infty$ for some $p>J_{F}^{+}+1$, then it holds uniformly for all $t \in \Lambda$ that

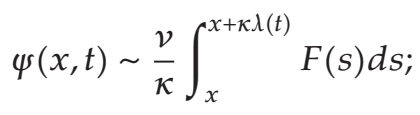


(iii) if $F \in \mathcal{R}_{-\alpha}, \bar{G}(x) \sim c \bar{F}(x)$ for some constant $c>0$, and $E \theta_{1}^{p}<\infty$ for some $p>\alpha+1$, then it holds uniformly for all $t \in \Lambda$ that

$$
\psi(x, t) \sim \frac{v+c \mu^{\alpha}}{\kappa} \int_{x}^{x+\kappa \lambda(t)} F(s) d s .
$$

Recently, Zhang et al. [3] extended the results of Aleškevičienè et al. [2] to the case that the claim sizes $\left\{X_{i}^{(n)}, i \geq 1\right\}$ caused by $n$th accident are negatively associated and obtained a unified form of $\psi(x, t)$ as follows: let $\bar{F}(x) \sim c \bar{G}(x)$ for $c \in[0, \infty]$, and one of the conditions below holds: (i) for $c=0, G \in \mathcal{C}$, and $E \theta_{1}^{p}<\infty$ for some $p>J_{G}^{+}+1$; (ii) for $c>0, F \in \mathcal{C}$, and $E \theta_{1}^{p}<\infty$ for some $p>J_{F}^{+}+1$; then it holds uniformly for all $t \in \Lambda$ that

$$
\psi(x, t) \sim \frac{1}{\mathcal{\kappa}} \int_{x}^{x+\kappa \lambda(t)}\left(v F(s)+\bar{G}\left(\frac{s}{\mu}\right)\right) d s .
$$

Observe the results of Zhang et al. [3] especially extended case (iii) of Aleškevičienè et al. [2]. Also, Lin and Shen [4] considered a generalized compound renewal risk model with $\left\{X_{i}^{(n)}, i \geq 1\right\}$ satisfying one type of asymptotically quadrant subindependent structure and also obtained the same relations (2.2), (2.3), and (2.4) as that of Aleškevičienè et al. [2].

Inspired by the above results, we will further discuss some issues as follows:

(1) to cancel the moment condition on $\left\{\theta_{i}, i \geq 1\right\}$, namely, $E \theta_{1}^{p}<\infty$ for some $p>J_{G}^{+}+1$, and $E \theta_{1}^{p}<\infty$ for some $p>J_{F}^{+}+1$;

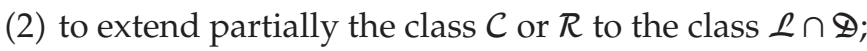

(3) to discuss the case when $\left\{X_{i}^{(n)}, i \geq 1\right\}$ are WUOD and $\left\{\theta_{i}, i \geq 1\right\}$ are WLOD;

(4) to drop the interrelationships between $\bar{F}$ and $\bar{G}$ and investigate the case when both $F$ and $G$ are heavy tailed.

In the paper, we will answer the four issues directly, and then we obtain our main results in the next section.

\subsection{Main Results}

For the main results of this paper, we now state some conditions which are that of Wang et al. [9].

Condition 1. The interarrival times $\left\{\theta_{i}, i \geq 1\right\}$ are NLOD r.v.s.

Condition 2. The interarrival times $\left\{\theta_{i}, i \geq 1\right\}$ are WOD r.v.s and there exists a positive and nondecreasing function $g(x)$ such that $g(x) \uparrow \infty, x^{-k} g(x) \downarrow$ for some $0<k<1, E \theta_{1} g\left(\theta_{1}\right)<\infty$, and $\max \left\{g_{U}(n), g_{L}(n)\right\} \leq g(n)$ for all $n \geq 1$, where $x^{-k} g(x) \downarrow$ means that $x_{1}^{-k} g\left(x_{1}\right) \geq C x_{2}^{-k} g\left(x_{2}\right)$ for all $0 \leq x_{1}<x_{2}<\infty$ and some finite constant $C>0$.

Condition 3. The interarrival times $\left\{\theta_{i}, i \geq 1\right\}$ are WOD r.v.s with $E \theta_{1}^{p}<\infty$ for some $2 \leq p<\infty$ and there exists a constant $\alpha>0$ such that $\lim _{n \rightarrow \infty} \max \left\{g_{U}(n), g_{L}(n)\right\} n^{-\alpha}=0$. 
Condition 4. The interarrival times $\left\{\theta_{i}, i \geq 1\right\}$ are WOD r.v.s with $E e^{\beta \theta_{1}}<\infty$ for some $0<\beta<\infty$ and $\lim _{n \rightarrow \infty} \max \left\{g_{U}(n), g_{L}(n)\right\} e^{-\gamma n}=0$ for any $\gamma>0$.

The first main result of this paper is the following.

Theorem 2.1. Consider the generalized compound renewal risk model with Assumptions $H_{1}^{*}, H_{2}^{*}$, and $\mathrm{H}_{3}$ and condition (1.7), there exists a finite constant $\alpha>0$ such that

$$
\lim _{n \rightarrow \infty} g_{L}(n) n^{-\alpha}=0, \quad \lim _{n \rightarrow \infty} g_{U}(n) e^{-\gamma n}=0, \quad \text { for any } \gamma>0
$$

Meanwhile, let one of Conditions 1-4 hold, and one of Conditions 1-4 with $\left\{\theta_{i}, i \geq 1\right\}$ replaced by $\left\{X_{i}^{(n)}, i \geq 1\right\}$ still holds.

(1) If $\bar{F}(x) \sim c \bar{G}(x)$ for some $c \in[0, \infty]$, additionally, for $c=0$ and $G \in \mathcal{C}$; for $0<c<\infty$ and $F \in \mathcal{C}$; for $c=\infty$ and $F \in \mathcal{L} \cap \Phi$, then relation (2.4) holds uniformly for all $t \in \Lambda$.

(2) If $F \in \mathcal{L} \cap \Phi$ and $G \in \mathcal{C}$, relation (2.4) holds uniformly for all $t \in \Lambda$.

Note that there do exist some WLOD r.v.s satisfying condition (2.5), see Wang et al. [19]. In the second main result below, we discuss the random time ruin probability, which requires another assumption.

\section{Assumption $\mathrm{H}_{4}$}

Let $\tau$ be nonnegative r.v. and independent of the sequences $\left\{\theta_{i}, i \geq 1\right\},\left\{X_{i}^{n}, i \geq 1, n \geq 1\right\}$, and $\left\{N_{n}, n \geq 1\right\}$.

Define $\Delta=\{\tau: P(\tau \in \Lambda)>0\}$.

Theorem 2.2. Under conditions of Theorem 2.1 and Assumption $\mathrm{H}_{4}$, one has

(1) if $\bar{F}(x) \sim c \bar{G}(x)$ for some $c \in[0, \infty]$, additionally, for $c=0$ and $G \in \mathcal{C}$; for $0<c<\infty$ and

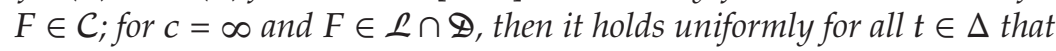

$$
\psi(x, \tau) \sim \frac{1}{\mathcal{K}} E \int_{x}^{x+\kappa \lambda(\tau)}\left(v F(s)+\bar{G}\left(\frac{s}{\mu}\right)\right) d s
$$

(2) if $F \in \mathcal{L} \cap \Phi$ and $G \in \mathcal{C}$, then relation (2.6) still holds uniformly for all $t \in \Delta$.

Remark 2.3. According to the proofs below of Theorems 2.1 and 2.2, we can see that Conditions $1-4$ are doing nothing more than making $\left\{\theta_{i}, i \geq 1\right\}$ and $\left\{X_{i}^{n}, i \geq 1\right\}$ satisfy the strong law of large number, namely,

$$
\lim _{k \rightarrow \infty} k^{-1} \sum_{i=1}^{k} \theta_{i}=\lambda^{-1}, \quad \lim _{k \rightarrow \infty} k^{-1} \sum_{i=1}^{k} X_{i}^{(n)}=\mu \quad \text { a.s.. }
$$

So, Conditions 1-4 in Theorems 2.1 and 2.2 can be replaced by (2.7). 


\section{Proofs of Main Results}

In this section we will give the proofs of our main results, for which we need some following lemmas.

Lemma 3.1. If $\left\{\xi_{i}, 1 \leq i \leq n\right\}$ are $n$ WUOD and nonnegative r.v.s with distributions $V_{i} \in \mathcal{L} \cap \Phi, 1 \leq$ $i \leq n$, respectively, then for any fixed $0<a \leq b<\infty$,

$$
P\left(\sum_{i=1}^{n} c_{i} \xi_{i}>x\right) \sim \sum_{i=1}^{n} P\left(c_{i} \xi_{i}>x\right)
$$

holds uniformly for all $\underline{c_{n}}=\left(c_{1}, c_{2}, \ldots, c_{n}\right) \in[a, b]^{n}$.

Proof. See Lemma 3.1 of Gao et al. [25].

Particularly, let $c_{1}=c_{2}=\cdots c_{n}=1$ in Lemma 3.2, we have a lemma below.

Lemma 3.2. If $\left\{\xi_{i}, 1 \leq i \leq n\right\}$ are $n$ WUOD and nonnegative r.v.s with distributions $V_{i} \in \mathcal{L} \cap \Phi, 1 \leq$ $i \leq n$, then

$$
P\left(\sum_{i=1}^{n} \xi_{i}>x\right) \sim \sum_{i=1}^{n} \bar{V}_{i}(x) .
$$

Lemma 3.3. If $\left\{\xi_{i}, i \geq 1\right\}$ are WUOD and real-valued r.v.s with common distribution $V \in \Phi$ and mean 0 and satisfying

$$
\lim _{n \rightarrow \infty} g_{U}(n) e^{-\gamma n}=0 \quad \text { for any } \gamma>0,
$$

then for any $\gamma>0$, there exists a constants $C=C(\gamma)$ such that

$$
P\left(\sum_{i=1}^{n} \xi_{i}>x\right) \leq C n \bar{V}(x)
$$

holds for all $x \geq \gamma n$ and all $n \geq 1$.

Proof. By Proposition 1.2 and along the same lines of the proof of Theorem 3.1 of Tang [26] with slight modifications, we can derive that, for some positive integer $m$,

$$
\sup _{n \geq m, x \geq \gamma n} \frac{P\left(\sum_{i=1}^{n} \xi_{i}>x\right)}{n \bar{V}(x)}<\infty
$$


From Lemma 3.2, we have

$$
\sup _{1 \leq n \leq m, x \geq \gamma n} \frac{P\left(\sum_{i=1}^{n} \xi_{i}>x\right)}{n \bar{V}(x)} \leq \sum_{n=1}^{m} \sup _{x \geq \gamma n} \frac{P\left(\sum_{i=1}^{n} \xi_{i}>x\right)}{n \bar{V}(x)}<\infty .
$$

Combining (3.5) and (3.6), there exists a constant $C(\gamma)>0$ such that (3.4) holds for all $x \geq \gamma n$ and all $n \geq 1$.

The following lemma discusses the strong law of large numbers for widely dependent r.v.s, which is due to Wang and Cheng [27].

Lemma 3.4. Let $\left\{\xi_{i}, i \geq 1\right\}$ be a sequence of real-valued r.v.s with finite mean $a>0$ and satisfy one of the Conditions $1-4$ with $\left\{\theta_{i}, i \geq 1\right\}$ replaced by $\left\{\xi_{i}, i \geq 1\right\}$. Then

$$
\lim _{n \rightarrow \infty} n^{-1} \sum_{i=1}^{n} \xi_{i}=a \quad \text { a.s.. }
$$

Proof. Follow Theorem 1 of Matula [28], Theorem 1.4, and the proofs of Theorems 1.1 and 1.2 of Wang and Cheng [27], respectively.

The lemma below gives the tail behavior of random sum, which extends the results of Aleškevičienè et al. [2] and Zhang et al. [3].

Lemma 3.5. Let $\left\{\xi_{i}, i \geq 1\right\}$ be a sequence of identically distributed and real-valued r.v.s with distribution $V$ and finite mean $a$ and satisfy one of the Conditions $1-4$ with $\left\{\theta_{i}, i \geq 1\right\}$ replaced by $\left\{\xi_{i}, i \geq 1\right\}$, where for Conditions 2 and 3 one further assumes that (3.3) holds. Let $\eta$ be nonnegative integer-valued r.v. with distribution $U$ and finite mean $b$, independent of $\left\{\xi_{i}, i \geq 1\right\}$. Assume that $\bar{V}(x) \sim c \bar{U}(x)$ for some $c \in[0, \infty]$.

(1) If $0 \leq c<\infty, U \in \mathcal{C}$, and the conditions of Lemma 3.4 are valid, then

$$
P\left(\sum_{i=1}^{\eta} \xi_{i}>x\right) \sim b \bar{V}(x)+\bar{U}\left(\frac{x}{a}\right)
$$

(2) If $c=\infty$ and $V \in \mathcal{\perp} \cap$, then relation (3.8) holds.

(3) Let no assumption be made on the interrelationship between $\bar{V}$ and $\bar{U}$. If $V \in \mathcal{L} \cap \Phi, U \in \mathcal{C}$, and the conditions of Lemma 3.4 are still valid, then relation (3.8) still holds.

Proof. Because $\eta$ has finite mean, there exists a large integer $m_{0}>0$ such that, for any fixed $\varepsilon>0$, it holds that

$$
E \eta \mathbf{1}_{\left\{\eta>m_{0}\right\}} \leq \varepsilon
$$


(1) First consider the case that $0<c<\infty$. Clearly, $U \in \mathcal{C}$ implies $V \in \mathcal{C}$. For any $x>0$ and any $\delta \in(0,1)$, we have

$$
\begin{aligned}
P\left(\sum_{i=1}^{\eta} \xi_{i}>x\right) & =\sum_{n=1}^{\infty} P\left(\sum_{i=1}^{n} \xi_{i}>x\right) P(\eta=n) \\
& =\left(\sum_{n=1}^{m_{0}}+\sum_{n=m_{0}+1}^{(1-\delta) x / a}+\sum_{n>(1-\delta) x / a}\right) P\left(\sum_{i=1}^{n} \xi_{i}>x\right) P(\eta=n) \\
& =K_{1}+K_{2}+K_{3} .
\end{aligned}
$$

For $K_{1}$, by Lemma 3.2 it follows that

$$
K_{1} \sim \bar{V}(x) \sum_{n=1}^{m_{0}} n P(\eta=n) \leq b \bar{V}(x)
$$

For $K_{2}$, since $n<(1-\delta) x / a$ and $V \in \mathcal{C} \subset \boldsymbol{\Phi}$, we obtain by Lemma 3.3 that

$$
\begin{aligned}
P\left(\sum_{i=1}^{n} \xi_{i}>x\right) & =P\left(\sum_{i=1}^{n}\left(\xi_{i}-a\right)>x-n a\right) \\
& \leq P\left(\sum_{i=1}^{n}\left(\xi_{i}-a\right)>\delta x\right) \\
& \leq C(\gamma) n \bar{V}(\delta x) \\
& \leq \widetilde{C}(\gamma) n \bar{V}(x),
\end{aligned}
$$

where $\gamma=\delta a /(1-\delta), C(\gamma)$ and $\tilde{C}(\gamma)$ are two constants only depending on $\gamma$. Hence, applying (3.9), Lemma 3.2, and the dominated convergence theorem can yield that

$$
K_{2} \sim \bar{V}(x) \sum_{n=m_{0}+1}^{(1-\delta) x / a} n P(\eta=n) \leq \bar{V}(x) E \eta \mathbf{1}_{\left\{\eta>m_{0}\right\}} \leq \varepsilon \bar{V}(x)
$$

For $K_{3}$, since $\delta \in(0,1)$ can be arbitrarily close to 0 , we see by $U \in \mathcal{C}$ that

$$
K_{3} \leq \bar{U}\left(\frac{(1-\delta) x}{a}\right) \lesssim \bar{U}\left(\frac{x}{a}\right)
$$

Substituting (3.11)-(3.14) into (3.10) and considering the arbitrariness of $\varepsilon>0$, we derive that

$$
P\left(\sum_{i=1}^{\eta} \xi_{i}>x\right) \lesssim b \bar{V}(x)+\bar{U}\left(\frac{x}{a}\right)
$$


On the other hand, we note that

$$
\begin{aligned}
P\left(\sum_{i=1}^{\eta} \xi_{i}>x\right) & =\sum_{n=1}^{\infty} P\left(\sum_{i=1}^{n} \xi_{i}>x\right) P(\eta=n) \\
& \geq\left(\sum_{n=1}^{m_{0}}+\sum_{n>(1+\delta) x / a}\right) P\left(\sum_{i=1}^{n} \xi_{i}>x\right) P(\eta=n) \\
& =K_{1}+K_{4} .
\end{aligned}
$$

For $K_{1}$, by Lemma 3.2 and (3.9), we get

$$
K_{1} \sim\left(b-E \eta \mathbf{1}_{\left\{\eta>m_{0}\right\}}\right) \bar{V}(x) \geq(b-\varepsilon) \bar{V}(x) .
$$

For $K_{4}$, by Lemma 3.4 we find that

$$
\lim _{n \rightarrow \infty} P\left(\frac{\sum_{i=1}^{n} \xi_{i}}{n}-a>-\frac{a \delta}{1+\delta}\right)=1
$$

which, along with $V \in \mathcal{C}$ and the arbitrariness of $\delta \in(0,1)$, leads to

$$
\begin{aligned}
K_{4} \geq & \sum_{n>(1+\delta) x / a} P\left(\frac{\sum_{i=1}^{n} \xi_{i}}{n}-a>-\frac{a \delta}{1+\delta}\right) P(\eta=n) \\
& \gtrsim \bar{U}\left(\frac{(1+\delta) x}{a}\right) \gtrsim \bar{U}\left(\frac{x}{a}\right) .
\end{aligned}
$$

Hence, from (3.16)-(3.19) and the arbitrariness of $\varepsilon>0$, we obtain that

$$
P\left(\sum_{i=1}^{\eta} \xi_{i}>x\right) \gtrsim b \bar{V}(x)+\bar{U}\left(\frac{x}{a}\right) .
$$

So, combining (3.15) and (3.20) proves that (3.8) holds for $0<c<\infty$.

Next we turn to the case that $c=0$, namely, $\bar{V}(x)=o(\bar{U}(x))$. According to Lemma 4.4 of Faÿ et al. [29], there exists a nondecreasing slowly varying function $L(x) \rightarrow \infty$ such that $\bar{V}(x)=o(\bar{U}(x) / L(x))$, which results in that for some $x_{0}>0$,

$$
\bar{V}(x) \leq \bar{U}(x) / L(x) \leq 1, \quad \text { for all } x \geq x_{0}
$$


Define

$$
\begin{gathered}
\overline{V_{*}}(x)= \begin{cases}\frac{1,}{\bar{U}(x)}, & \text { if } 0 \leq x<x_{0}, \\
\frac{L(x)}{} & \text { if } x \geq x_{0},\end{cases} \\
V_{*}^{-1}(y)=\inf \left\{x \in(-\infty, \infty): V_{*}(x) \geq y\right\}, \quad 0 \leq y \leq 1,
\end{gathered}
$$

where $V_{*}(x)=1-\overline{V_{*}}(x)$. Let

$$
\xi_{i}^{*}=V_{*}^{-1}\left(V\left(\xi_{i}\right)\right), \quad i \geq 1
$$

It is easy to verify that $\left\{\xi_{i}^{*}, i \geq 1\right\}$ are still WUOD and identically distributed r.v.s with common distribution $V_{*} \in \mathcal{C}$. By the definition of $\overline{V_{*}}$, we know that $\xi_{i} \leq \xi_{i}^{*}, i \geq 1$, and then $a \leq E \xi_{i}^{*}=$ $a^{*}<\infty$. Thus, $K_{1}+K_{2}$ in (3.10) is divided into three parts as

$$
\begin{aligned}
K_{1}+K_{2} \leq & \left(\sum_{n=1}^{m_{0}}+\sum_{n=m_{0}+1}^{(1-\delta) x / a^{*}}\right) P\left(\sum_{i=1}^{n} \xi_{i}^{*}>x\right) P(\eta=n) \\
& +\sum_{(1-\delta) x / a^{*}<n \leq(1-\delta) x / a} P\left(\sum_{i=1}^{n} \xi_{i}>x\right) P(\eta=n) \\
= & K_{1}^{\prime}+K_{2}^{\prime}+K_{2}^{\prime \prime} .
\end{aligned}
$$

Clearly, $\left\{\xi_{i}^{*}, i \geq 1\right\}$ are such that the conditions of Lemmas 3.2 and 3.3 hold, then (3.11) and (3.13) can still hold with $K_{1}, K_{2}$, and $\left\{\xi_{i}, i \geq 1\right\}$ replaced by $K_{1}^{\prime}, K_{2}^{\prime}$, and $\left\{\xi_{i}^{*}, i \geq 1\right\}$. So, we deduce by $V_{*} \in \mathcal{C} \subset \Phi$ and $\overline{V_{*}}(x)=o(\bar{U}(x))$ that

$$
K_{1}^{\prime}+K_{2}^{\prime} \lesssim(b+\varepsilon) \cdot \frac{\overline{V_{*}}(x)}{\overline{V_{*}}(x / a)} \cdot \frac{\overline{V_{*}}(x / a)}{\bar{U}(x / a)} \cdot \bar{U}\left(\frac{x}{a}\right)=o\left(\bar{U}\left(\frac{x}{a}\right)\right) .
$$

For $K_{2}^{\prime \prime}$, it follows from Lemma 3.4 that

$$
\lim _{n \rightarrow \infty} P\left(\frac{\sum_{i=1}^{n} \xi_{i}}{n}-a>\frac{a \delta}{1-\delta}\right)=0 .
$$

Then, by $U \in \mathcal{C} \subset \Phi$ we have

$$
\begin{aligned}
K_{2}^{\prime \prime} & \leq \sum_{(1-\delta) x / a^{*}<n \leq(1-\delta) x / a} P\left(\frac{\sum_{i=1}^{n} \xi_{i}}{n}-a>\frac{a \delta}{1-\delta}\right) P(\eta=n) \\
& =o\left(\bar{U}\left(\frac{(1-\delta) x}{a^{*}}\right)\right)=o\left(\bar{U}\left(\frac{x}{a}\right)\right) .
\end{aligned}
$$


From (3.10), (3.14), and (3.24)-(3.27), we find that

$$
P\left(\sum_{i=1}^{\eta} \xi_{i}>x\right) \lesssim \bar{U}\left(\frac{x}{a}\right)
$$

Again by (3.16) and (3.19), it is seen that

$$
P\left(\sum_{i=1}^{\eta} \xi_{i}>x\right) \geq K_{4} \gtrsim \bar{U}\left(\frac{x}{a}\right)
$$

Since $U \in \mathcal{C} \subset \Phi$ and $\bar{V}(x)=o(\bar{U}(x))$, we get

$$
\bar{V}(x)=\frac{\bar{V}(x)}{\bar{U}(x)} \cdot \frac{\bar{U}(x)}{\bar{U}(x / a)} \cdot \bar{U}\left(\frac{x}{a}\right)=o\left(\bar{U}\left(\frac{x}{a}\right)\right) .
$$

Consequently, we obtain by combining (3.28)-(3.30) that (3.8) holds for $c=0$.

(2) Now we deal with the case that $c=\infty$, namely, $\bar{U}(x)=o(\bar{V}(x))$. Apparently, when $V \in \mathcal{L} \cap \Phi$, we can derive by Lemmas 3.2 and 3.3 that (3.11) and (3.13) still hold. As for $K_{3}$, by $\bar{U}(x)=o(\bar{V}(x))$ and $V \in \mathcal{L} \cap \Phi$, we know that

$$
K_{3} \leq \frac{\bar{U}((1-\delta) x / a)}{\bar{V}((1-\delta) x / a)} \cdot \frac{\bar{V}((1-\delta) x / a)}{\bar{V}(x)} \cdot \bar{V}(x)=o(\bar{V}(x)) .
$$

Substituting (3.11), (3.13), and (3.31) into (3.10) implies that

$$
P\left(\sum_{i=1}^{\eta} \xi_{i}>x\right) \lesssim b \bar{V}(x) .
$$

For $V \in \mathcal{L} \cap \Phi$, by Lemma 3.2 we also get (3.17). As for $K_{4}$, arguing as (3.19) and (3.31), we still have that

$$
K_{4} \gtrsim \bar{U}\left(\frac{(1+\delta) x}{a}\right)=o(\bar{V}(x))
$$

From (3.16), (3.17), and (3.33), we conclude that

$$
P\left(\sum_{i=1}^{\eta} \xi_{i}>x\right) \gtrsim b \bar{V}(x) .
$$

Similarly to the derivation of (3.30), by $\bar{U}(x)=o(\bar{V}(x))$ and $V \in \mathcal{L} \cap \Phi$ we still see that $\bar{U}(x / a)=o(b \bar{V}(x))$. This, along with (3.32) and (3.34), gives relation (3.8) immediately. 
(3) According to the proof of (2), we know that if $V \in \mathcal{L} \cap \Phi$, then (3.11) and (3.13) hold. While from the proof of (1), we have (3.14) if $U \in \mathcal{C}$. Hence, under the conditions of (3), we obtain (3.15).

On the other hand, from the proof of (2), we can get (3.17) when $V \in \mathcal{L} \cap \Phi$. Again by the proof of (1), relation (3.19) also holds for $U \in \mathcal{C}$. So, (3.20) is proved under the conditions of (3). As a result, we show (3.8) directly.

The next two lemmas will give some results of the renewal risk model, which is the compound renewal risk model with $N_{1}=N_{2}=\cdots=1$.

Condition 5. For $\alpha$ in (2.5), there exist $t_{0} \in \Lambda \cap(0, \infty)$ and $f$ in (1.12) such that $(f(x))^{\alpha}\left(P\left(\theta_{1} \leq\right.\right.$ $\left.\left.t_{0}\right)\right)^{f(x)}=o(\bar{F}(x))$.

Lemma 3.6 (Corollary 2.1 of Wang et al. [9]). Consider the compound renewal risk model with $N_{1}=N_{2}=\cdots=1$ and $c>\lambda \mu$, in which $\left\{X_{i}, i \geq 1\right\}$ are i.i.d.r.v.s with common distribution $F$, and $\left\{\theta_{i}, i \geq 1\right\}$ are WLOD r.v.s satisfying (2.5) and one of Conditions 1-4.

(1) If $F \in \mathcal{S}_{*}$, then for any $t_{0} \in \Lambda$, it holds uniformly for all $t \in\left(t_{0}, \infty\right)$ that

$$
\psi(x, t) \sim \frac{\lambda}{c-\lambda \mu} \int_{x}^{x+\mu \lambda(t)} \bar{F}(s) d s
$$

(2) Furthermore, if Condition 5 holds, then relation (3.35) still holds uniformly for all $t \in \Lambda$.

Lemma 3.7 (Corollary 2.2 of Wang et al. [9]). Under conditions of Lemma 3.6 and assumption $\mathrm{H}_{4}$, one has

(1) if $F \in \mathcal{S}_{*}$, then

$$
\psi(x, \tau) \sim \frac{\lambda}{c-\lambda \mu} E \int_{x}^{x+\mu \lambda(\tau)} \bar{F}(s) d s
$$

holds uniformly for all $\tau \in\left\{\tau: P\left(\tau I_{\left\{\tau \geq t_{0}\right\}} \in \Lambda\right)>0\right\}$ for any $t_{0} \in \Lambda$.

(2) Additionally, if Condition 5 holds, then relation (3.36) still holds uniformly for all $t \in \Delta$.

Now we prove the main results as follows.

Proof of Theorem 2.1. Clearly, if $F \in \mathcal{L} \cap \Phi$ then $F$ satisfies Condition 5. In fact, the assumption $F \in \mathcal{L} \cap \Phi$ indicates that the tail of $F$ behaves essentially like a power function, thus there exists $q>0$ such that $x^{q} \bar{F}(x) \rightarrow \infty$. Take $f(x)=x^{p}$ for any $0<p<1$, which satisfies (1.12). So, for any $s>0, e^{-s x^{p}} / \bar{F}(x) \rightarrow 0$ and Condition 5 holds.

First, consider Theorem 2.1(1). For the case that $0 \leq c<\infty$, we can obtain relation (2.4) by (1.5) and Lemmas 3.5(1) and 3.6, and going along the similar ways to that of 
Theorems 2.2(2) and (3) of Aleškevičienè et al. [2]. For the case that $c=\infty$, since $F \in \mathcal{L} \cap \boldsymbol{\Phi}$, by Lemma 3.5(2) we have that for any $y>0$,

$$
\limsup _{x \rightarrow \infty} \frac{P\left(S_{N}>x y\right)}{P\left(S_{N}>x\right)}=\limsup _{x \rightarrow \infty} \frac{\bar{F}(x y)}{\bar{F}(x)}<\infty, \quad \lim _{x \rightarrow \infty} \frac{P\left(S_{N}>x+y\right)}{P\left(S_{N}>x\right)}=\lim _{x \rightarrow \infty} \frac{\bar{F}(x+y)}{\bar{F}(x)}=1,
$$

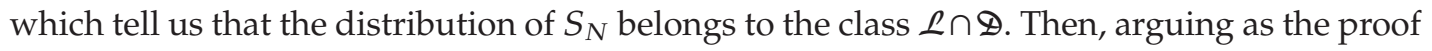
of Theorem 2.2(1) of Aleškevičienè et al. [2], we also obtain relation (2.4).

Now consider Theorem 2.1(2). By Lemma 3.5(3), $F \in \mathcal{L} \cap \Phi$ and $G \in \mathcal{C}$, it is also clear that the distribution of $S_{N}$ belongs to $\mathcal{L} \cap \boldsymbol{\Phi}$. Hence, relation (2.4) still holds from (1.5) and Lemmas 3.5(3) and 3.6.

Proof of Theorem 2.2. By (1.6), the uniformity of (2.4) in Theorem 2.1, and the independence between $\tau$ and the risk system, we can get the proof of Theorem 2.2.

\section{Acknowledgments}

The authors would like to thank Professor Yuebao Wang for his thoughtful comments and also thank the editor and the anonymous referees for their very valuable comments on an earlier version of this paper. The work is supported by Research Start-up Foundation of Nanjing Audit University (no. NSRC10022), Natural Science Foundation of Jiangsu Province of China (no. BK2010480), and Natural Science Foundation of Jiangsu Higher Education Institutions of China (no. 11KJD110002).

\section{References}

[1] Q. Tang, C. Su, T. Jiang, and J. Zhang, "Large deviations for heavy-tailed random sums in compound renewal model," Statistics E Probability Letters, vol. 52, no. 1, pp. 91-100, 2001.

[2] A. Aleškevičienè, R. Leipus, and J. Šiaulys, "Tail behavior of random sums under consistent variation with applications to the compound renewal risk model," Extremes, vol. 11, no. 3, pp. 261-279, 2008.

[3] J. Zhang, F. Cheng, and Y. Wang, "Tail behavior of random sums of negatively associated increments," Journal of Mathematical Analysis and Applications, vol. 376, no. 1, pp. 64-73, 2011.

[4] Z. Lin and X. Shen, "Approximation of the tail probability of dependent random sums under consistent variation and applications," Methodology and Computing in Applied Probability. In press.

[5] Yang, Y. Wang K, and J. Liu, "Asymptotics and uniform asymptotics for finite-time and infinite-time absolute ruin probabilities in a dependent compound renewal risk model," Journal of Mathematical Analysis and Applications, vol. 398, no. 1, pp. 352-361, 2013.

[6] Q. Tang, "Asymptotics for the finite time ruin probability in the renewal model with consistent variation," Stochastic Models, vol. 20, no. 3, pp. 281-297, 2004.

[7] R. Leipus and J. Šiaulys, "Asymptotic behaviour of the finite-time ruin probability under subexponential claim sizes," Insurance: Mathematics E Economics, vol. 40, no. 3, pp. 498-508, 2007.

[8] Y. Yang, R. Leipus, J. Šiaulys, and Y. Cang, "Uniform estimates for the finite-time ruin probability in the dependent renewal risk model," Journal of Mathematical Analysis and Applications, vol. 383, no. 1, pp. 215-225, 2011.

[9] Y. Wang, Z. Cui, K. Wang, and X. Ma, "Uniform asymptotics of the finite-time ruin probability for all times," Journal of Mathematical Analysis and Applications, vol. 390, no. 1, pp. 208-223, 2012.

[10] V. P. Chistyakov, "A theorem on sums of independent positive random variables and its applications to branching processes," Theory of Probability and Its Applications, vol. 9, pp. 640-648, 1964. 
[11] D. A. Korshunov, "Large deviation probabilities for the maxima of sums of independent summands with a negative mean and a subexponential distribution," Theory of Probability and Its Applications, vol. 46, no. 2, pp. 355-366, 2001.

[12] W. Feller, "One-sided analogues of Karamata's regular variation," L'Enseignement Mathématique, vol. 15, pp. 107-121, 1969.

[13] D. B. H. Cline, "Intermediate regular and П variation," Proceedings of the London Mathematical Society, vol. 68, no. 3, pp. 594-616, 1994.

[14] D. B. H. Cline and G. Samorodnitsky, "Subexponentiality of the product of independent random variables," Stochastic Processes and their Applications, vol. 49, no. 1, pp. 75-98, 1994.

[15] C. Klüppelberg, "Subexponential distributions and integrated tails," Journal of Applied Probability, vol. 25, no. 1, pp. 132-141, 1988.

[16] P. Embrechts, C. Klüppelberg, and T. Mikosch, Modelling Extremal Events for Insurance and Finance, vol. 33 of Applications of Mathematics, Springer, Berlin, Germany, 1997.

[17] D. Denisov, S. Foss, and D. Korshunov, "Tail asymptotics for the supremum of a random walk when the mean is not finite," Queueing Systems, vol. 46, no. 1-2, pp. 15-33, 2004.

[18] N. H. Bingham, C. M. Goldie, and J. L. Teugels, Regular Variation, vol. 27 of Encyclopedia of Mathematics and its Applications, Cambridge University Press, Cambridge, UK, 1987.

[19] K. Wang, Y. Wang, and Q. Gao, "Uniform asymptotics for the finite-time ruin probability of a dependent risk model with a constant interest rate," Methodology and Computing in Applied Probability. In press.

[20] N. Ebrahimi and M. Ghosh, "Multivariate negative dependence," Communications in Statistics A, vol. 10, no. 4, pp. 307-337, 1981.

[21] H. W. Block, T. H. Savits, and M. Shaked, "Some concepts of negative dependence," The Annals of Probability, vol. 10, no. 3, pp. 765-772, 1982.

[22] L. Liu, "Precise large deviations for dependent random variables with heavy tails," Statistics $\mathcal{E}$ Probability Letters, vol. 79, no. 9, pp. 1290-1298, 2009.

[23] Y. Chen, K. C. Yuen, and K. W. Ng, "Precise large deviations of random sums in presence of negative dependence and consistent variation," Methodology and Computing in Applied Probability, vol. 13, no. 4, pp. 821-833, 2011.

[24] K. Joag-Dev and F. Proschan, "Negative association of random variables, with applications," The Annals of Statistics, vol. 11, no. 1, pp. 286-295, 1983.

[25] Q. Gao, P. Gu, and N. Jin, "Asymptotic behavior of the finite-time ruin probability with constant interest force and WUOD heavy-tailed claims," Asia-Pacific Journal of Risk and Insurance, vol. 6, no. 1, 2012.

[26] Q. Tang, "Insensitivity to negative dependence of the asymptotic behavior of precise large deviations," Electronic Journal of Probability, vol. 11, no. 4, pp. 107-120, 2006.

[27] Y. Wang and D. Cheng, "Basic renewal theorems for random walks with widely dependent increments," Journal of Mathematical Analysis and Applications, vol. 384, no. 2, pp. 597-606, 2011.

[28] P. Matuła, "A note on the almost sure convergence of sums of negatively dependent random variables," Statistics E Probability Letters, vol. 15, no. 3, pp. 209-213, 1992.

[29] G. Faÿ, B. González-Arévalo, T. Mikosch, and G. Samorodnitsky, "Modeling teletraffic arrivals by a Poisson cluster process," Queueing Systems. Theory and Applications, vol. 54, no. 2, pp. 121-140, 2006. 


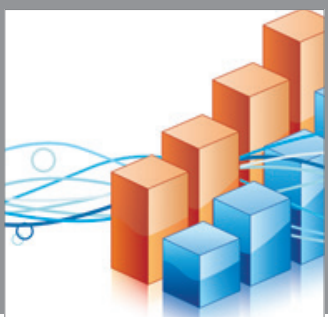

Advances in

Operations Research

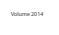

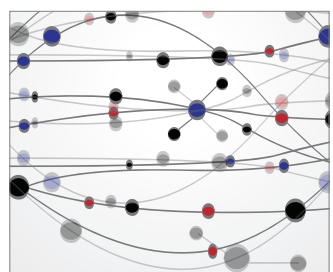

\section{The Scientific} World Journal
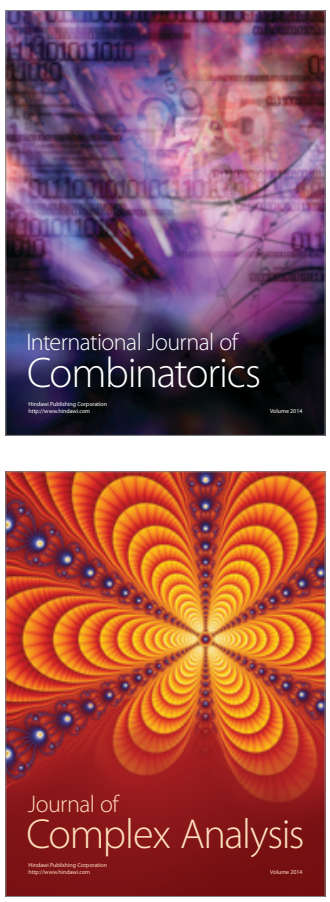

International Journal of

Mathematics and

Mathematical

Sciences
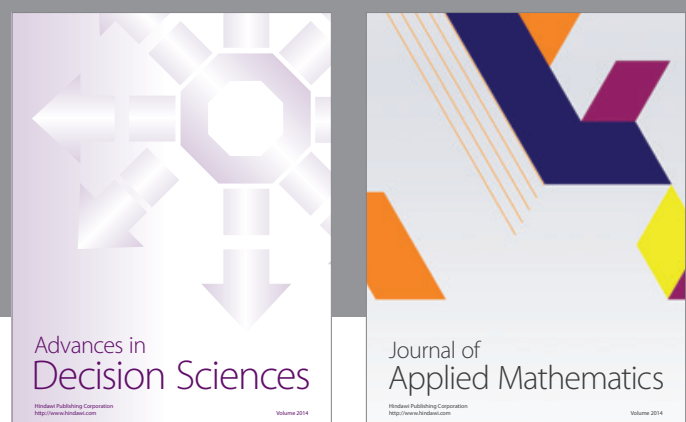

Journal of

Applied Mathematics
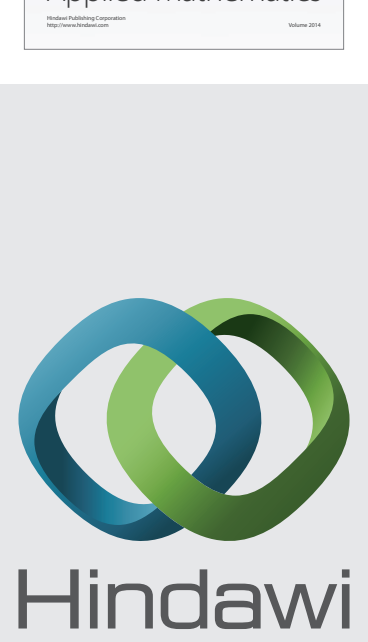

Submit your manuscripts at http://www.hindawi.com
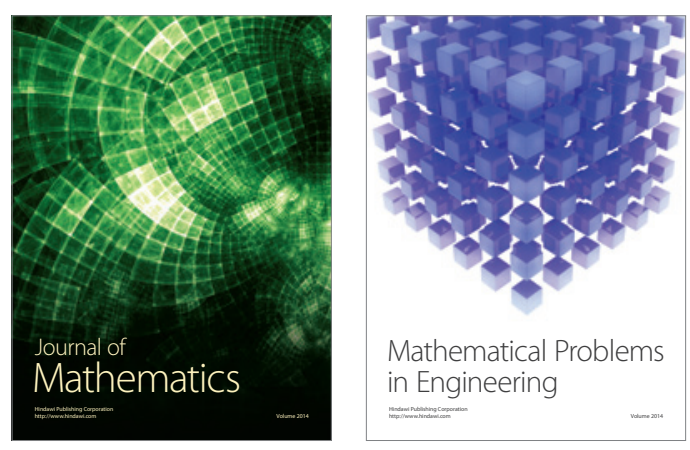

Mathematical Problems in Engineering
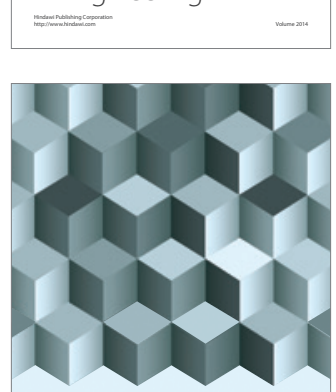

Journal of

Function Spaces
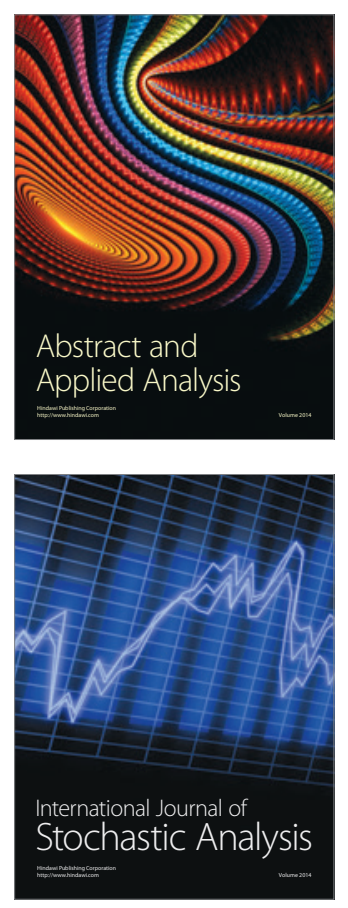

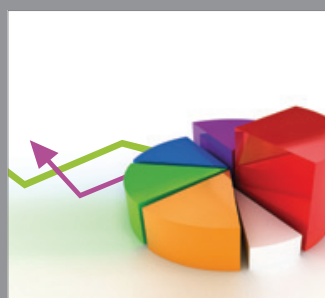

ournal of

Probability and Statistics

Promensencen
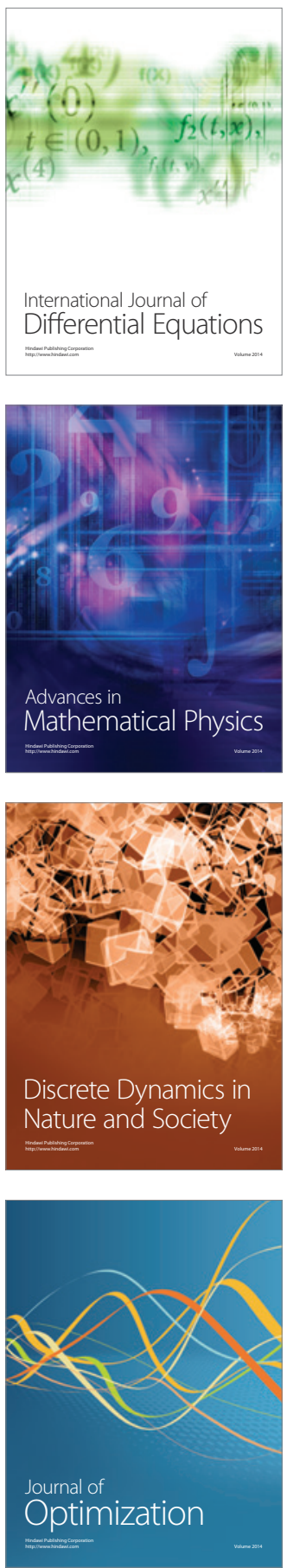